\title{
Donna - A web based Al Personal Assistant
}

\author{
Ami Doshi \\ Computer Engineering \\ K.J Somaiya COE \\ Mumbai
}

\author{
Ria Shah \\ Computer Engineering \\ K.J Somaiya COE \\ Mumbai
}

\author{
Drasti Bhimani \\ Computer Engineering \\ K.J Somaiya COE \\ Mumbai
}

\author{
Bhoomi Patel \\ Computer Engineering \\ K.J Somaiya COE \\ Mumbai
}

\author{
Swati Mali \\ Mentor \\ Department of Computer Engineering \\ K. J. Somaiya COE \\ Mumbai
}

\begin{abstract}
In a world that is moving so swiftly and where everyone is driven towards making even the unimaginable a reality, the idea of making the use of artificial intelligence to turn mankind's desires into reality has secured a place into every individual's mindset. This project 'Donna' aims to turn the desire of a common man to have a personal assistant for himself into reality. Donna is an artificial intelligence based personal assistant that can be used by the simplest of individuals to get their daily tasks and upcoming schedules fixed according to their own convenience. The process is automated in a way such that any work or meeting related mail to the user is automatically forwarded to Donna and the whole back and forth job of getting the meeting fixed at a date and time that is convenient to both the user and the client is done completely by her. A task manager is also provided in the interface that can add and delete tasks for the user and displays the daily tasks as well the upcoming events sorted based on priority and deadlines. The user can also have quick chat with
\end{abstract}

Donna through the chatbot that is provided which is also integrated with the text to speech feature in an attempt to make Donna more user friendly for any user.

\section{Keywords}

AI, Personal Assistant, Task Manager, Chatbot

\section{INTRODUCTION}

Nowadays, planning has become an integral part of everyone's life. Most of the times, planning ends up taking more of a person's time than the actual implementation. This has given rise to whole new set of professionals called the personal assistants whose job is to plan schedules and meetings efficiently of the person who has hired him. Although most of the people have a busy schedule and need an assistant to plan their daily tasks, not every individual can afford to have a personal secretary for themselves.

This project, Donna, aims at creating a personal assistant based on artificial intelligence that can plan schedules and meetings of the user. Whenever a mail regarding an appointment or a meeting is sent to the user, it is automatically forwarded to Donna who does the whole back and forth job of getting the meeting fixed at a date and time that is convenient to both the sender as well as the user. For this task to be carried out successfully, Donna is integrated with the google calendar of the user, through which it can make sure that the user is free on that particular date and time. A chatbot is also created along with the interface through which the user can talk to Donna and also later see the conversation logs.

\section{BACKGROUND / LITERATURE SURVEY}

\subsection{Chatbot}

Turing test acts as a criterion for intelligence, whose article was published by Alan Turing in 1950. The article describes the criterion to be the ability of a computer program to impersonate a human in a real-time written conversation with a judge in such a way that the judge is unable to distinguish whether it is a program or a real human who is in conversation with him

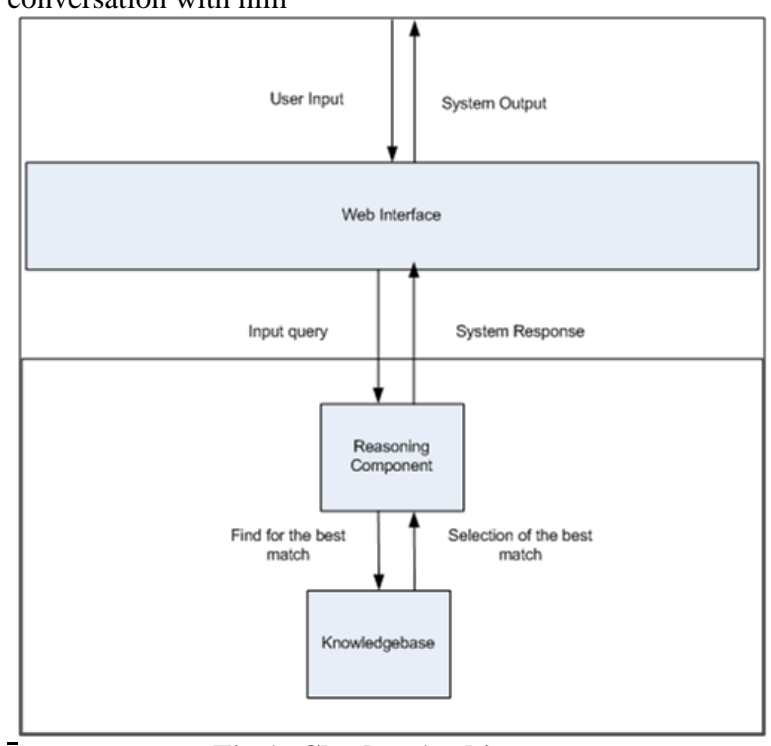

Fig 1: Chatbot Architecture

The components of basic chatbot would include a knowledge base, an inference system and a user interface through which the user can communicate with the bot. Knowledge base is used for storing the data and the rules. To generate the appropriate output, an inference system matches the input with the data and rules present in the knowledge base. The knowledge base as well the inference engines should be dependent on the type of chatbot being created and would always differ from system to system. A chatbot can be web based or standalone. 


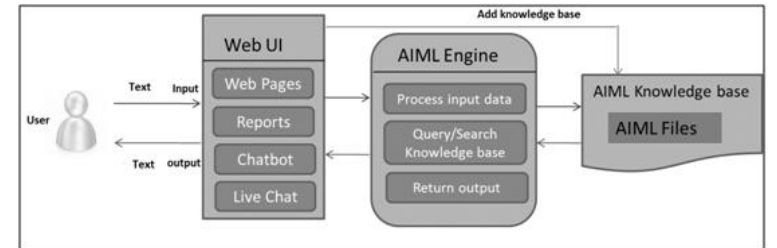

Fig 2: Basic Architecture of a PATTERN MATCHING AIML CHATBOT

Different applications of chatbot are now becoming a reality due to the awareness created among the people about a chatbot and its distinguished uses. Various chatbots are being created having varied personalities and purposes.

\subsection{Personal Assistant}

\subsubsection{Capturing Common Knowledge about}

Tasks: Intelligent Assistance for To-Do Lists: ${ }^{[1]}$

To-do lists are a prevalent form of task management. The paper focuses of three aspects for a smart assistance: use of intelligent agents in a office setting; paraphrase-based approach to match agent capabilities with the to-dos; and use for personal tasks along with the types of assistance that can be provided to the user by elaborating them on the basis of knowledge extracted from the Web. Lastly, it explores the coordination between different users via a to-do management application deployed on Social Networks.

\subsubsection{PTIME: Personalized Assistance for Calendaring $^{[2]}$}

As the world is moving towards electronics calendars, a personalized assistance for calendar becomes the need of the hour. PTIME (Personalized Assistance for Calendaring) is a smart and predictive assistant which helps managing tasks like meetings requests, reserve venues and schedule events. It is adaptive and flexible in nature, learning from its history of prefered schedules. It is user-friendly and allows user to collaborate with non PTIME users.

\subsection{Existing System: \\ 2.3.1 A.L.I.C.E. ${ }^{[3]}$}

When talks on are about chatbots that works on Pattern Matching Strategy, one of the first one to be talked about is Artificial Linguistic Internet Computer Entity (A.L.I.C.E.). It is widely used chatbot that is open source which contains AIML files covering wide range of fields. These fields have been included to the knowledge base of Donna along with another category "Meeting" of its own design. This file aims to cover various queries related to the meetings as Donna concentrates more towards the scheduling module.

\subsubsection{X.ai $(A M Y)^{[4]}$}

$\mathrm{X}$.ai has come up with one of the advanced personal assistant with a chatbot named "Amy". It is still in its Beta Version providing a free version which is provided to users after a wait of more than a year and a paid version. A user has to CC (send a carbon copy) of the email to AMY (amy@x.ai). Then, Amy talks to the other party, sets up a preferable meeting timing, and then sends an acknowledgement emails to both the parties. The idea to create Donna was inspired by Amy. Donna is better than Amy in a way that emails have to be forwarded as a carbon copy to Amy; whereas Donna intelligently extracts emails which are related to meetings from the user's account. As Amy is still in its Beta version, it is not possible to compare its working and its features with Donna.

\section{PROPOSED SYSTEM}

\subsection{Comparison of Existing System}

This project, Donna is mainly created to provide a tool based on artificial intelligence to the user that fixes meetings and schedule of the user through email communication. The whole process is automated and the calendar of the user is integrated with Donna to have a complete idea of the user's existing schedules. Donna also aims at solving conflicts, in case any, based on the user's priority. Also, the system has an interface where the user can talk to the bot, teach it and see conversation logs.

Table 1: Comparison of Existing AI Assistants

\begin{tabular}{|c|c|c|c|c|}
\hline Name & Developer & Origin & Functions & Gaps Identified \\
\hline Siri & Apple & $\begin{array}{r}\text { Perhaps the first virtual } \\
\text { personal assistant to enter } \\
\text { the mainstream, } \\
\text { 'Siri'debuted on the } \\
\text { iPhone in } 2011 .\end{array}$ & $\begin{array}{r}\text { With the arrival of iOS } 10, \text { Siri } \\
\text { widely opened to third-party apps } \\
\text { allowing developers outside } \\
\text { Apple to give it capabilities such } \\
\text { as hailing an Uber if you simply } \\
\text { ask for one which in itself is a } \\
\text { pinnacle of achievement }\end{array}$ & $\begin{array}{r}\text { Criticized early on for poor } \\
\text { speech recognition since } \\
\text { Apple was behind rivals in } \\
\text { "neural network" technology, } \\
\text { Apple has improved this } \\
\text { technology recently. }\end{array}$ \\
\hline Alexa & Amazon & $\begin{array}{r}\text { Alexa is the name } \\
\text { Amazon gave the digital } \\
\text { assistant living inside its } \\
\text { Echohome device, which } \\
\text { started selling widely in } \\
\text { June } 2015\end{array}$ & $\begin{array}{l}\text { Alexa began with limited uses, } \\
\text { most often responding from the } \\
\text { in-home speaker to request for } \\
\text { things like weather and news. It } \\
\text { will take commands like } \\
\text { remembering your shopping list. }\end{array}$ & $\begin{array}{r}\text { Alexa isn't in your phone; it's } \\
\text { stuck inside the Echo or the } \\
\text { newer Dot device. Amazon } \\
\text { just made it available in its } \\
\text { tablets. }\end{array}$ \\
\hline Cortana & Microsoft & $\begin{array}{l}\text { Cortana was introduced } \\
\text { in 2014, named after the } \\
\text { AI character in the hit } \\
\text { Xbox game "Halo." }\end{array}$ & $\begin{array}{l}\text { Cortana a 'digital agent' can } \\
\text { handle basics like controlling } \\
\text { calendars, getting weather and } \\
\text { taking dictation on an email. }\end{array}$ & $\begin{array}{r}\text { Cortana is only as smart as its } \\
\text { programming, and it gets } \\
\text { much of its information from } \\
\text { Microsoft's Bing. It does not } \\
\text { use Google, the search leader }\end{array}$ \\
\hline
\end{tabular}




\begin{tabular}{|c|c|c|c|c|}
\hline Assistant & Google & $\begin{array}{r}\text { In 2012, Google added } \\
\text { Google Now to phones. } \\
\text { Google's AI advanced } \\
\text { with Google Home, and } \\
\text { Allo, both bearing a } \\
\text { voice-commanded helper } \\
\text { known as Google } \\
\text { Assistant. }\end{array}$ & $\begin{array}{r}\text { Google Assistant acts like Siri or } \\
\text { Cortana, as an assistant that can } \\
\text { call up information and perform } \\
\text { tasks like turning on Spotify and } \\
\text { giving directions. Also, Google } \\
\text { taps into its own knowledge graph } \\
\text { to retrieve the relevant } \\
\text { information for users. }\end{array}$ & $\begin{array}{l}\text { Google gets higher scrutiny on } \\
\text { the Privacy issue because of } \\
\text { all the data it has. It's going to } \\
\text { be a challenge to balance } \\
\text { privacy with always-on. ever- } \\
\text { listening digital assistance. }\end{array}$ \\
\hline
\end{tabular}

\subsection{Our Functionalities to Overcome the Above Shortcomings}

\subsubsection{Good Text to speech}

In an attempt to make the chatbot more user friendly, text to speech is implemented in the chatbot. On writing a message to Donna, she replies in text as well as in speech to the user.

\subsubsection{Web based}

Since the personal assistant is web based, it is publicly available to the user. Therefore, the user does not need to buy the personal assistant and can use all of its features free of cost.

\subsubsection{Checks calendar}

The user's calendar is integrated with Donna so that Donna can automatically check the user's calendar and set meetings according to the convenience of the user. It also takes into consideration weekends and public holidays and sets meetings based on priority.

\subsubsection{Uses AIML}

It makes use of AIML files to generate smart and logical replies to the user.

\subsection{Architecture}

The entire Architecture is divided into two parts:

a. Generation of mails

1. Whenever User receives an email with subject as 'Appointment' or 'meeting', the mail is automatically sent to DONNA, the personal Assistant.

2. The mail so received is parsed and is checked with AIML database.

3. The parsed mail also checks the Google Calendar to obtain the User's schedule.

4. With the help of Google Calendar, a suitable timing is generated and with the help of AIML files, a suitable reply is generated.

5. Both these replies are merged and the output file is sent to mail server which is further forwarded to the client.

\section{b. Learning component}

With the help of learning agent, Donna will generate Templates for Calendar Update and will also generate new AIML templates which are fed to Google Calendar database and AIML database respectively. 


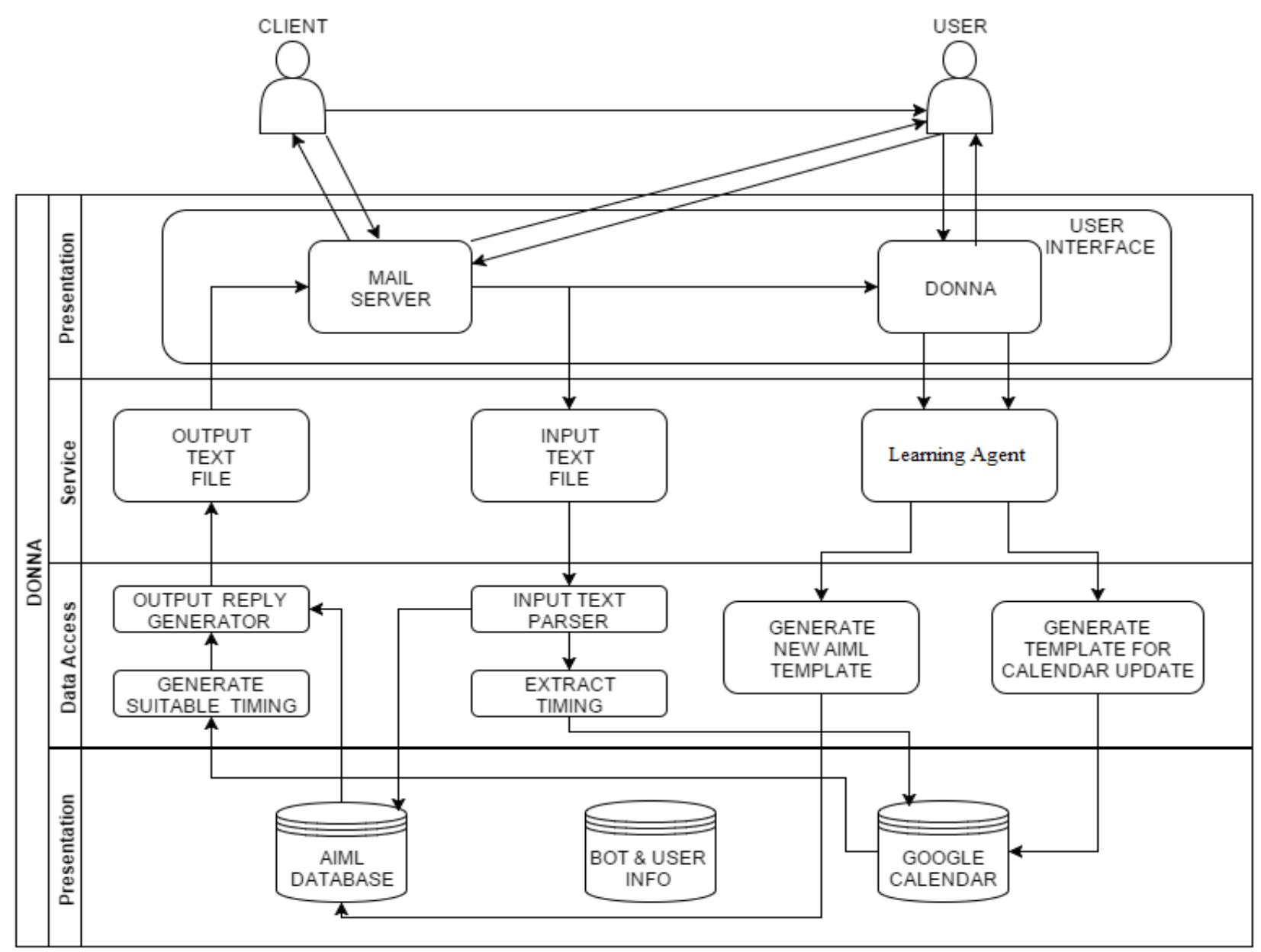

Fig 3: System Architecture

\section{EVALUATION AND ANALYSIS}

\subsection{Chatbot}

A chatbot is a computer program designed to simulate an intelligent conversation with a human user via auditory or textual methods. Most of the chat-bots are designed for engaging in small talk and their personalities are created by the programmer. Chat-bots are designed to make humans into thinking that they are talking to a human, but instead they are talking to a machine. Taking advantage of this transparency property of chat-bot, an artificial character and personality can be given to a chat-bot which acts like a person of a specific profession.

Designing chat-bots using the current state of the art, mainly uses rules written in AIML (Artificial Intelligence Markup Language) or ChatScript.

Program-O is a good open source software which allows one to easily design a chatbot.

The files generated are in AIML format and easily modifiable. Since Donna acts as a personal assistant and focuses on meeting, a module has been added targeting "meetings". Along with it, it can answer questions related various fields.

\section{What would you like to know?}

\section{what s sour name

Fig 4: Question asked to chatbot

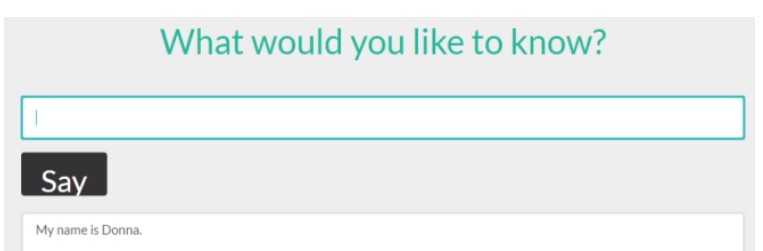

Fig 5: Reply obtained from the chatbot

\begin{tabular}{|c|c|c|}
\hline Show $10 \cdot$ entries & & Search: L \\
\hline Date & Client & Donna \\
\hline 2017-03-2500:30:07 & WHAT IS YOUR NAME & My name is Donna. \\
\hline 2017-03-23 14:23:45 & DO YOU KNOW HARRY POTTER & is a bestselling author. \\
\hline 2017-03-23 14:23:26 & WHAT IS YOUR NAME & My name is Donna. \\
\hline
\end{tabular}

\subsection{Text-to-Speech ${ }^{[9]}$}

Fig 6: Chat History

In an attempt to make the chatbot more user friendly, another feature that is added to the chatbot in Donna is Text-to-Speech (TTS). For android applications, it can be easily inculcated but Donna being a web-based agent required some efforts. For the implementation of text to speech in the chatbot, an open source software called Voice RSS is used. It provides a unique key that needs to be added to the php file of the API. The code consists of different parameters through which the rate as well the accent of the speech can be modified. All these functionalities in the chatbot makes it more easy to use for the user.

Parameters used: 
- API key: Generated from the API and used in the PHP code.

- $\quad$ src: The textual content for converting text to speech.

- $\quad$ hl: The textual content language.

- $\quad r$ The speech rate

\subsection{Email replying}

The automation in email replying is done following two approaches:

$\circ \quad$ Where the user wants to initiate the meeting.

- Where the user receives an email regarding the meeting.

\section{User initiates meeting}

This is a part where the USER wants to initiate a meeting and send an appointment request to the client. It is the simplest part of the entire working system. The user has to fill a form containing the details of Client's name, Client's email id, the date and time of meeting, and the reason for the appointment.

\section{Get the receiver's details}

The form collects all the data entered by the USER, converts it into a text format and sends it to the receiver.

\section{Form and send the mail}

Donna collects all the information from the form and creates a file converting the details into an Email format with the subject being set as "Regarding an Appointment". It stores the Email address of the client into a different variable and uses it to send the file to the client.

\section{User receives meeting message}

\section{Pass the email to the chatbot}

As soon as the user receives any mail from the client, it is forwarded it to Donna.

\section{Read the email}

When Donna receives the mail, it checks for the top UNREAD mails received. It will select the first unread mail, read the contents of the mail and stores it into a text file followed by passing it for parsing.

\section{Pattern matching}

Pattern matching is a type of parsing in which the system searches for keywords in a sentence and based on that keywords, it searches the database for a match and replies with the corresponding answer. This system uses an open source software Program-O that uses a pattern matching algorithm for reading and generating a reply.

The system reads the content of the mail from a text file. It will separate the E-mail ID of the client required later for the reply.

\section{Check the calendar}

Donna while parsing the Email, reads the condition of the meeting sent by the client. Using Google Calendar API, Donna checks the user's calendar whether he is free for the particular time slot of that day and accordingly generates a reply.

\section{Reply Generation}

As discussed earlier, Donna stores the email-iD of the client so as to send the generated reply to the corresponding Email address. After parsing the mail, system has generated a reply for a sentence which is stored in a text file say REPLY. It repeats the process of parsing the sentence and generation a reply, and appends the output to the file.

\section{Send the email}

After the process terminates, using Gmail API, Donna sends the REPLY text file in an Email format to the client.

\section{Update calendar}

As soon as an appointment is scheduled for the user, Donna immediately updates user's Google Calendar with the following meeting and blocks the slot as Meeting. Ultimately, it sends an invitation to both the parties i.e. the user as well as the client.

The various mail actions are done with help of PHPMailer, Php and MySql.

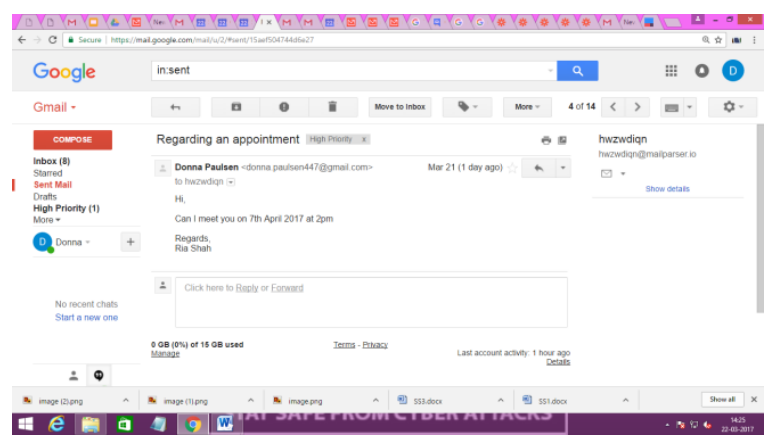

Fig 7: Reply from Donna through email

\subsection{Calendar}

The user's Google calendar is integrated with Donna so that Donna can set up meetings for the user considering the most convenient time for him. The main task here was to parse emails and extract the date and time of the meeting that is proposed by the client to the user. This task was completed by using Mailparser.io ${ }^{[8]}$. And then Zapier ${ }^{[6]}$ was used to integrate the Google calendar and the parsed mail and also to create an event on the date and time that was mentioned in the email which is reflected in the user's Google calendar . The steps for setting up given as follows:

- $\quad$ Mail parsed using mailparser.io ${ }^{[8]}$ and dates and time extracted using parsing rules.

- An event created in Harvey's calendar on the date and time that is extracted from the client's mail.

- $\quad Z_{a p i e r}{ }^{[6]}$ is used for connecting the parsed mail of Donna to the Google calendar of Harvey.

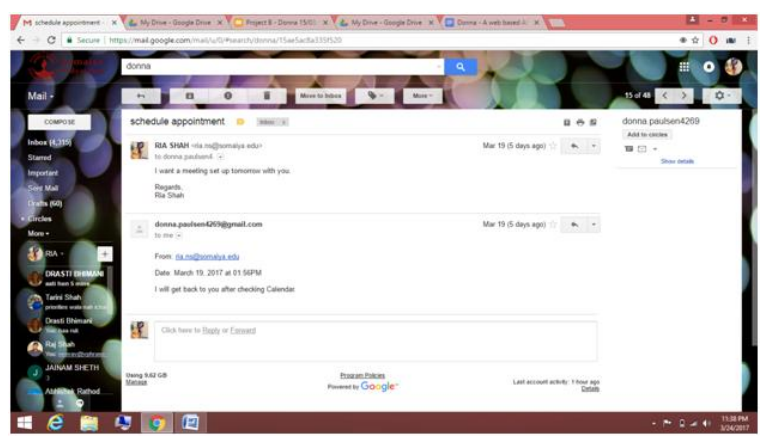

Fig 8: Scheduling a meeting 


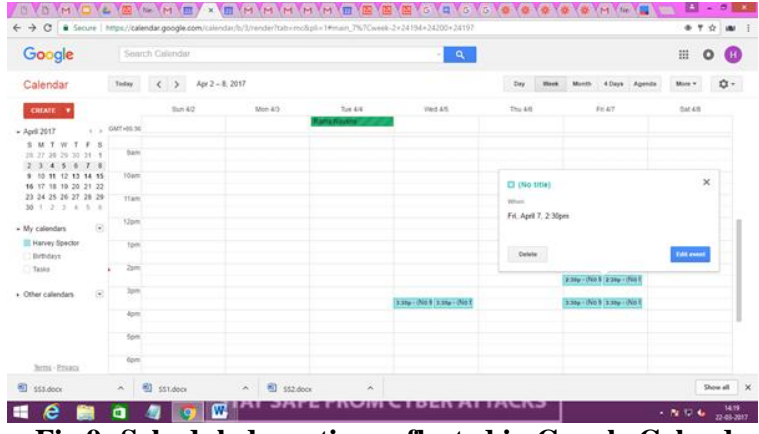

Fig 9: Scheduled meeting reflected in Google Calendar

\subsection{Task Manager ${ }^{[5]}$}

Today, an average person leads a hectic life. Daily numerous tasks are to be completed, some are daily whereas some are weekly. Completing them on time that too efficiently and in a time saving manner is a challenge.

Donna, being a smart assistant helps out with maintaining the various tasks to be done and also gives an optimised schedule as a suggestion to complete all the tasks efficiently.

It does the following tasks:

- Creating tasks: Task is added by the user with name, priority and deadline.

- Upcoming Tasks: All the upcoming tasks are shown sorted on the basis of their deadline date.

- Delete Task: A task can be deleted.

- Schedule Task: It shows the suggested schedule of the tasks. Steps for the same are:

Step 1: Sort deadline(ascending) and priority(descending)

Step 2:

CalculatedPriority $==\left(3 *^{\prime}\right.$ Priority ${ }^{\prime}+5 *(367-D e$ adline))

- arbitrary chosen to reflect the rules that:

A-Due Date is the Most Important Factor

- B-Period Category is the next most important factor

Step 3 : Integrating the java code with UI

- Constraints/Assumptions that are considered:

- Each task contains a name, start date, priority and a deadline.

- Time constraints (start date should not be prior to the current date)

○ Current parameters: Deadline and Priority.
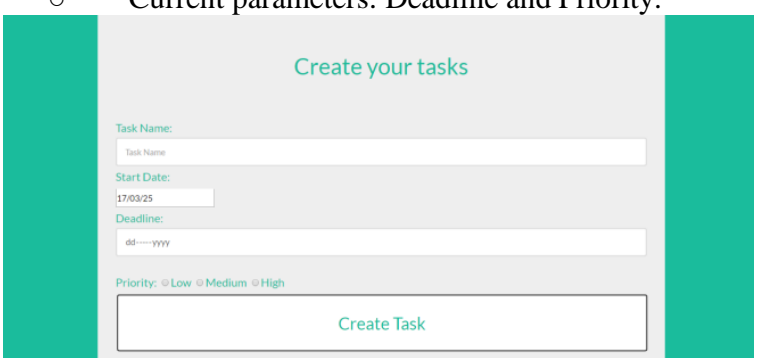

Fig 10: Create task
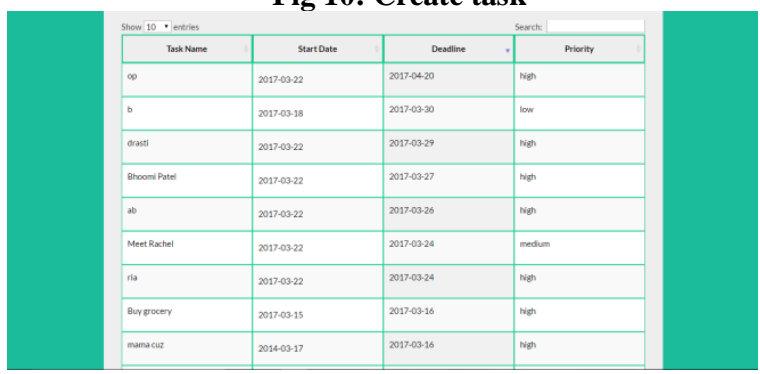

Fig 11: View Upcoming tasks

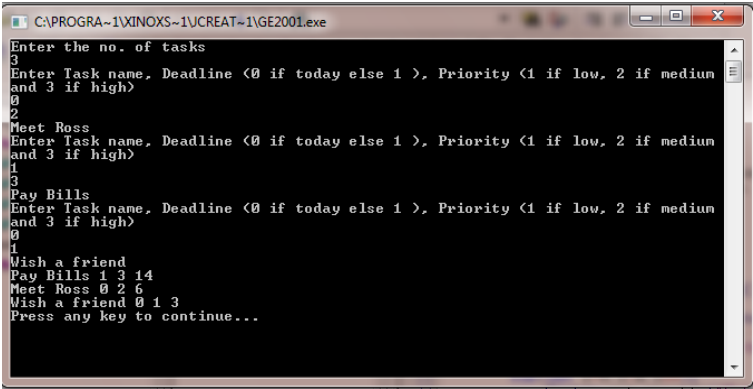

Fig 12: Schedule Created

\section{LIMITATIONS}

1. The AI Assistant does not take into consideration travelling locations i.e. for example, if meeting $\mathrm{A}$ is scheduled in New York at 13:00 for 1 hour and meeting B is scheduled in New Jersey at 14:00, the AI Assistant will check the calendar and schedule the meeting without considering the time required to travel from New York to New Jersey.

2. For checking logs and daily tasks, one will always have to $\log$ in to their account by visiting the website.

3. Currently, the system is built by as if it is solely used by one user. Hence, it does not have any features such as log in and $\log$ out

4. Currently, the assistant does not deal with multiple clients regarding the same meeting

\section{CONCLUSION}

Thus, with this software we hope to save everyone's time, as we are all aware of the platitude; Time is Money. Also, the bot provides other features to the user like chatting and learning. Hence, being one of the best implementations of Artificial Intelligence, the software is a combination of assistance, usefulness and entertainment.

\section{REFERENCES}

[1] Yolanda Gil, Varun Ratnakar, Timothy Chklovski, Paul Groth, Denny Vrandecic Capturing Common Knowledge about Tasks: Intelligent Assistance for To-Do Lists"

[2] Pauline M. Berry, Melinda Gervasio, Bart Peintner, Neil Yorke-Smith "PTIME: Personalized Assistance for Calendaring"

[3] A.L.I.C.E. Artificial Intelligence Foundation, "https://alice.pandorabots.com"

[4] X.ai, "https://x.ai"

[5] Task Manager, "http://math.stackexchange.com"

[6] Zapier, "https://zapier.com/app/explore"

[7] IFTTT "https://ifttt.com/discover"

[8] MailParser.io "https://app.mailparser.io/account/login/?red=myaccount \%2Fsubscription"

[9] Text-to-speech "http://www.voicerss.org/sdk/php.aspx" 